\title{
Molecular Weight, Osmotic Second Virial Coefficient, and Extinction Coefficient of Colloidal CdSe Nanocrystals
}

\author{
A. Striolo, J. Ward, J.M. Prausnitz* \\ Chemical Engineering Department, University of California, Berkeley, and \\ Chemical Sciences Division, Lawrence Berkeley National Laboratory, \\ Berkeley, CA 94720 \\ and \\ W.J. Parak, D. Zanchet, D. Gerion, D. Milliron, and A. P. Alivisatos \\ Chemistry Department, University of California, Berkeley, and \\ Material Sciences Division, Lawrence Berkeley National Laboratory, \\ Berkeley, CA 94720
}

\begin{abstract}
Membrane osmometry is used to measure osmotic pressures of dilute solutions containing quasi-spherical CdSe nanocrystals covered with polymer brushes in toluene in the range 31 to $45^{\circ} \mathrm{C}$. Osmotic-pressure data, as a function of nanocrystal concentration, yield the molecular weight and the osmotic second virial coefficient of the nanocrystals; the latter is related to the potential of mean force between two nanocrystal particles in dilute solution.

Coupled with molecular-weight data, extinction coefficients and oscillator strengths are also obtained for nanocrystals of various sizes in toluene. CdSe nanocrystal sizes were obtained either from Transmission Electron Microscopy or from correlations between the wavelength of the absorbing peak and nanocrystal size.

Osmotic-pressure data are reduced with a simple perturbed hard-sphere equation of state; the perturbation is due to long-range (London dispersion) attraction and a shortrange interaction potential. The only adjustable parameter, the strength of this short-range potential, shows two-body repulsion or attraction, depending on the sample and on solution conditions.
\end{abstract}

Key words: CdSe Nanocrystals, Molecular Weight, Second Osmotic Virial Coefficient

* Author to whom correspondence should be addressed 


\section{Introduction}

Semiconductor clusters, containing hundreds or a few thousand atoms, exhibit strongly size-dependent optical and electrical properties. Current research is focused on the individual properties of quantum dots as well as on their collective behavior; joining the dots into complex assemblies creates opportunities for synthesizing new materials [1]. For example, elongated CdSe nanocrystals show polarized luminescent emission along their long axis, unlike spherical dots, which emit plane-polarized light [2]. CdSe nanocrystals can be prepared in a wide range of shapes, including rods, teardrops, etc. by changing surfactant composition and monomer concentration during their synthesis [3]. By applying the strategies developed to synthesize CdSe quantum dots, Puntes et al. [4] produced magnetic cobalt nanorods as well as spherically shaped nanocrystals.

Semiconductor nanocrystals may be used as fluorescent labels [5]. When coupled to bio-molecules, nanocrystals may serve as ultra-sensitive biological detectors [6]. However, the nature of the ligand can significantly influence the photophysics of the particles [7]. When nanocrystals are incorporated into complex nano-structures, it is of increasing importance to know their molecular weight to control the assembly process. The relationship between the molar adsorption coefficient and nanocrystal size is also of fundamental importance.

In recent years, CdSe particles have become a model system in nanocrystal research, including the effect of quantum confinement on optical properties. The literature $[8,9]$ provides a relationship between particle diameter and the absorption spectra for CdSe nanocrystals. Schmelz et al. [10] recently showed that the molar extinction coefficient at the first exciton peak increases approximately with the third power of the particle diameter.

To date, however, no experimental measurements have been reported to relate the molecular weight of a nanocrystal to its size. In this work, membrane osmometry is used to measure the molecular weight and the osmotic second virial coefficient of quasispherical CdSe nanocrystals with diameters in the range 2 to $6 \mathrm{~nm}$. We also report the effective two-body potential of mean force for pairs of nanocrystals in dilute solution. This potential is useful for calculating phase diagrams that can serve as a guide for forthcoming applications of nanocrystal technology [11-13]. 


\section{Materials and Methods}

CdSe particles of different sizes were prepared via high-temperature organometallic chemistry [8,9]. A solution of dimethylcadmium and selenium powder in tributylphophine (TBP) was injected into liquid trioctylphosphine oxide (TOPO) at $360^{\circ} \mathrm{C}$. The desired particle size was achieved by varying the concentration of reactants, the reaction time, and by multiple injections. After some minutes growth at $300^{\circ} \mathrm{C}$, the reaction was stopped by cooling. The nanocrystals were precipitated by adding methanol. Following centrifugation, the supernatant was discarded and fresh methanol was added. An additional methanol wash removed residual TOPO molecules, ensuring that only surface-bound TOPO would be included in the samples. After vortexing for a few minutes, the new solution was again centrifuged and the supernatant was discarded. The precipitate was dried under $\mathrm{N}_{2}$ flow. By dissolving dried CdSe nanocrystal samples in toluene, solutions with controlled CdSe mass concentration $c_{m}[\mathrm{~g} / \mathrm{l}]$ were prepared.

Optical absorption spectra were recorded for all nanocrystal solutions of various mass concentrations. Fig. 1a shows a typical absorption spectrum. The mean absorption per mass concentration was calculated at the first absorption peak, $\lambda_{a b s}[\mathrm{~nm}]$, of the spectrum and combined with the molecular weight to find the molar extinction coefficient, $\Gamma(1 / \mathrm{mol}$ $\mathrm{cm}$ ) for each sample as shown in Table 1. To determine the integrated oscillator strength of the transition, the lower energy side of the first exciton peak was fit with a Gaussian on a linear energy scale. This procedure is necessary to compare the absorption strength of different-sized nanocrystals as the transition tends to narrow for larger sizes and widen for broader size distributions.

The nanocrystal's hard-core diameter $\sigma[\mathrm{nm}]$ was either directly measured using Transmission Electron Microscopy (TEM, FEI Tecnai $12120 \mathrm{kV}$ ) or by using an existing correlation between the wavelength of the absorbing peak and nanocrystals size [9]. Fig. $1 \mathrm{~b}$ shows a representative TEM image of a sample used in our experiments.

Osmotic pressures of nanocrystal solutions in toluene were measured with a Jupiter Inst. Co. Membrane Osmometer model 231 (Jupiter, Florida, USA) for several concentrations in the range 5 to $35 \mathrm{~g} / \mathrm{l}$. The cellulose-acetate membrane has a molecularweight cutoff at 10,000 g/mole. Solvent was purchased from Fisher and used as received. 
The osmotic pressure, $\Pi$, of a colloidal solution can be expressed by a virial expansion in the mass concentration (see, for example, Tombs and Peacoke [14]):

$\frac{\Pi}{R T}=\frac{1}{M_{n}} c_{m}+B c_{m}^{2}+\ldots$,

where $R, T, M_{n}, B$, and $c_{m}$ are, respectively, the gas constant, the absolute temperature, the nanoparticle number-average molecular weight, the osmotic second virial coefficient, and nanoparticle mass concentration. At low solute concentration, higher-order terms can be neglected.

Dilute solutions of CdSe nanocrystals were prepared in toluene and used shortly after preparation to avoid flocculation. For each temperature, fresh solutions were always prepared because when stored in toluene at room conditions CdSe nanocrystals tend to

flocculate, forming high-molecular-weight aggregates. Some test experiments were performed after dissolving a weighted amount of CdSe nanocrystals in mixtures containing toluene and five-to-ten volume percent excess TOPO.

$B$ and $M_{n}$ were obtained from fitting the experimental data to Eq.(1).

Fig. 2 shows a schematic representation of CdSe nanocrystals considered here. The semiconductor core is surrounded by polymer brushes.

\section{Results and Discussion}

\section{$\underline{\text { Molecular weight }}$}

Table 1 summarizes the experimental results for 19 different nanocrystals. As expected, the molecular weight rises with increasing nanocrystal diameter. Fig. 3 shows the experimental molecular weights as a function of nanocrystal diameter. The experimental uncertainty in molecular-weight determination is about $10-15 \%$. Molecular weights below 300,000-500,000 g/mole are determined with somewhat better accuracy. The experimental molecular weights are reasonably accurate for nanocrystal size less than 4-5 $\mathrm{nm}$. For higher sizes, membrane osmometry provides only a rough estimate of molecular weight. However, even for lower molecular weights, the results obtained here exceed previously reported molecular-weight calculations for nanocrystals. Test experiments for nanocrystals dissolved in toluene-TOPO solutions, not reported here for clarity, provided 
molecular weights as a function of nanocrystal diameter in agreement with those here presented.

As a first approximation, the nanocrystal molecular weight can be directly calculated from the hard-core diameter $\sigma$ and the density of the CdSe semiconductor bulk $(\delta=5.81$ $\mathrm{gcm}^{-3}$ ). High-resolution TEM images show that nanocrystals have the same structure as bulk CdSe, suggesting the same density $[9,15]$. The nanocrystal molecular weight is calculated by

$M_{n}=\frac{\pi}{6} N_{A} \delta \sigma^{3}$,

where $N_{A}$ is the Avogadro number. Results for these calculations are shown as the solid line in Fig. 3.

Because Eq.(2) considers monodisperse spherical nanocrystals, the calculated molecular weight scales with the nanocrystal volume, e.g. with the third power of the diameter. However, molecular weights calculated from Eq.(2) are significantly lower than those from osmometric data, especially for small nanocrystals. In a more realistic representation, the sample polydispersity should be accounted for. Instead of using one nominal diameter, it would be more realistic to use a Gaussian distribution of nanocrystal sizes around a mean diameter, as suggested by UV-vis spectra. However, the experimental and the calculated molecular weights cannot be reconciled by considering a realistic size distribution with a variance below $15 \%$.

Eq.(2) neglects the TOPO molecules adsorbed on the nanocrystal surface. A more realistic model should take into account the contribution of these molecules to the nanocrystal molecular weight. TOPO molecules cannot be resolved by TEM, because the contrast is too low; therefore, it is not possible to evaluate the increased CdSe nanocrystal diameter due to TOPO coverage. However, the additional molecular weight due to adsorbed molecules, $\Delta M_{n}$, should scale with the nanocrystal surface, i.e. with the second power of the nanocrystal diameter, according to:

$\Delta M_{n}=\beta M_{T O P O} \pi \sigma^{2}$.

In Eq.(3), $M_{\text {TOPO }}$ is the molecular weight of one TOPO molecule, $386.7 \mathrm{~g} / \mathrm{mol}$, and $\beta$ is a parameter that gives the number of TOPO molecules per unit surface area assuming uniform surface coverage. The broken line in Fig. 3 is obtained by adding the 
contribution due to TOPO surface coverage, Eq.(3), to the molecular weights calculated by Eq. (2). A surface coverage $\beta$ equal to $10 \mathrm{~nm}^{-2}$ was assumed.

TOPO molecules have a roughly conical shape. By calculating the number of conical TOPO molecules that could accommodate on a spherical CdSe nanocrystal, assuming that TOPO has a swept-out radius of $0.55 \mathrm{~nm}$ and a length of $0.99 \mathrm{~nm}$ [9], a value of $\beta$ can be calculated ranging from about 5 for smaller CdSe nanocrystals to about 2 for larger ones. However, Katari et al. [9] experimentally showed that the number of TOPO molecules bound to a CdSe nanocrystal is larger than that corresponding to this simple model, in agreement with our calculations.

When experimental uncertainty and sample polydispersity are taken into account, calculated (Eq.(2)+Eq.(3)) and experimental molecular weights agree reasonably well for a substantial surface coverage provided by TOPO molecules. Additional experimental uncertainty could be due to the presence of nanocrystal agglomerates in some samples; to the observation that smaller CdSe nanocrystals have larger percent TOPO coverage than larger nanocrystals [9]; or to the presence of unbound TOPO molecules on the nanocrystal surface.

\section{Osmotic second virial coefficient}

Fig. 4 shows experimental osmotic second virial coefficients as a function of nanocrystal diameter. Nanocrystals were in toluene solutions at approximately $38^{\circ} \mathrm{C}$ or $42^{\circ} \mathrm{C}$. Our results suggest that at $40 \pm 2^{\circ} \mathrm{C}$ the osmotic second virial coefficients decrease slightly with rising molecular size. However, more experimental data are required to draw a definite conclusion.

Fig. 5 shows experimental osmotic second virial coefficients in toluene as a function

of temperature. Nanocrystals considered here have molecular weights of approximately 170,000 or $500,000 \mathrm{~g} / \mathrm{mol}$ (see Table 1). Even though experimental uncertainty in $B$ is significant, our results suggest that $B$ increases with rising temperature. For $\mathrm{CdSe}$ nanocrystals covered with TOPO in toluene, our results indicate that the theta temperature (temperature at which $B$ is zero) is near $35^{\circ} \mathrm{C}$, although we have only few data below this temperature. When $B$ is positive, solute molecules tend to repel each other; when $B$ is negative, solute molecules tend to attract each other. 
Molecular extinction coefficient and oscillator strength

The oscillator strength of the first exciton transition in quantum dots is of fundamental interest and is closely related to the well-known blue shift of that transition with decreasing size. Recently, Schmelz, et al. [10] estimated the size-dependent absorption coefficient at the peak of this transition for CdSe nanocrystals. They found that the absorption coefficient increases approximately as the third power of the diameter, in linear proportion to the volume of the crystal. This result is equivalent to an oscillator strength (per CdSe unit) independent of particle size, which may correspond to the oscillator strength of bulk CdSe excitons. In contrast, results for CdS [16,17] and for CdTe [18] indicate that oscillator strength is enhanced for small particles, yielding approximately constant absorption per particle.

Our data qualitatively agree with those of Schmelz, et al. [10]: molar absorption for CdSe particles increases with size. Our results are based on the integrated absorption peak, thereby eliminating error associated with the changing width of the peak for different sizes and size distributions. This integration also allows comparison with the bulk oscillator strength. In bulk CdSe, the two lowest energy excitons have a similar oscillator strength near $5 \times 10^{-4}$ per CdSe unit. While our results indicate that CdSe nanocrystals have oscillator strengths in this range, they are not sufficiently plentiful to conclude that oscillator strength is size-independent. Our data weakly suggest a slight decrease in oscillator strength for smaller particles, in contrast to dramatic quantum enhancement for CdS and CdTe due to confinement [16-18].

\section{Osmotic Pressure from a Perturbed-Hard-Sphere Equation of State}

Nanocrystals are composed of a semiconductor hard core surrounded by adsorbed polymer brushes. For such systems, the potential of mean force can be approximated by a perturbed potential with a hard-sphere reference system and two perturbations: an attractive London dispersion potential, and a short-range interaction potential due to polymer brushes on the particle's surface. London dispersion forces decay with the sixth power of the inverse of the center-to-center separation. It is more difficult to specify the potential term arising from the adsorbed polymer brushes. The interaction between two 
flat polymer layers in a good solvent can be calculated as a repulsion decaying with the ninth power of the inverse of the separation between layers [19]. However, experimentally there is no unique characterization of the force profile between surfaces covered by adsorbed polymers. The force profile depends on surface coverage, solvent conditions, velocity of approach between the two surfaces, time allowed for the polymer molecules to adsorb on the surfaces, etc. [20]. In a first approximation, one could expect this interaction to be repulsive, due to the entropic effects of polymer overlap as the particles approach. However, in some cases, i.g. if the experimental temperature is below the theta temperature for the polymer brush, the expected short-range repulsion can become a short-range attraction [19-21]. A similar attraction could arise due to bridging of the polymer brush if the polymer brush density on the surface is low [22].

To represent this interaction, we simulated an interaction decaying with the ninth power of the inverse of the center-to-center separation between nanocrystals. The strength and sign of this interaction is used to fit the calculations to the experimental data.

Based on this simplified description, $W(r)$, the nanocrystal-nanocrystal effective potential of mean force is:

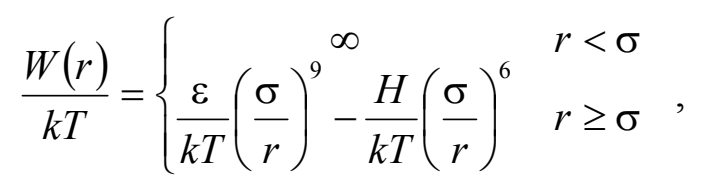

where $r$ is the center-to-center nanocrystal-nanocrystal distance; $\sigma$ is the nanocrystal diameter; $k$ is Boltzmann's constant; $H$ is Hamaker's constant and $\varepsilon$ is an adjustable parameter.

Bergstrom [23] computed the non-retarded Hamaker constant with the Lifshitz theory for several compounds. For cadmium sulfide in vacuum $H_{C d S}=11.010^{-20} \mathrm{~J}$. Bergstrom's value for $H_{C d S}$ in vacuum provides a good approximation for $\mathrm{H}_{\mathrm{CdSe}}$ toward calculating the effective Hamaker constant for CdSe in toluene. Non-retarded Hamaker constants for a few hydrocarbons were given by Israelachvili [21]. The average value is about $510^{-20} \mathrm{~J}$; we assume this value for toluene $\left(H_{t o l}\right)$. To obtain the Hamaker constant for nanocrystals in toluene, knowing $H_{C d S e}$ in vacuum and $H_{t o l}$, in vacuum, we apply the approximation proposed by Israelachvili [21]:

$$
H_{\text {eff }}=\left(\sqrt{H_{C d S}}-\sqrt{H_{t o l}}\right)^{2} \text {. }
$$


Using this approximation, the effective $H$ is $1.210^{-20} \mathrm{~J}$.

By applying the random-phase approximation (RPA), the osmotic-pressure data can be reproduced with a van der Waals-type equation of state. The reference state is the hardsphere system [24]; the perturbation is given by the effective nanocrystal-nanocrystal potential of mean force in Eq.(3). The osmotic pressure $\Pi$ depends on nanocrystal number density $\rho$ :

$$
\frac{\Pi}{\rho k T}=\frac{1+\eta+\eta^{2}-\eta^{3}}{(1-\eta)^{3}}+2 \pi \rho \cdot \int_{\sigma}^{\infty}\left\{1-\exp \left[-\frac{H}{k T}\left(\frac{\sigma}{r}\right)^{6}+\frac{\varepsilon}{k T}\left(\frac{\sigma}{r}\right)^{9}\right]\right\} \cdot r^{2} d r .
$$

In Eq.(6), $\eta$ is the packing fraction $\left(\eta=\frac{\pi}{6} \cdot \rho \cdot \sigma^{3}\right)$. Table 2 gives the parameters used to fit Eq.(6) to the experimental data. Here $\sigma$ is the particle diameter without adsorbed TOPO polymer. The only adjustable parameter is $\varepsilon$.

Because the experimental data are sometimes affected by possible nanocrystalnanocrystal coagulation, Eq.(6) does not always reproduce our osmotic-pressure measurements with meaningful values for parameter $\varepsilon$. Nevertheless, Fig. 7 shows reasonably good results for the nanocrystals described in Table 2. Results for adjustable parameter $\varepsilon$ indicate a strong short-range repulsion in two cases, and an additional shortrange attraction in three cases. The short-range repulsion can be explained by entropic effects due to overlap of the polymer brushes [19], while short-range attraction may be due to bridging of the polymer brushes [19], or to loss of TOPO coverage during sample preparation. For example, Mattousi et al. [22] found that smaller particles are more likely to present weak attractions due to lower surface coverage.

For simplicity, one could also reproduce the experimental osmotic-pressure data by setting $\varepsilon=0$ in Eq.(4) and by using $H$ as an adjustable parameter. For this latter case, the Hamaker constant is indicated by $H^{\prime}$. Table 2 gives values for $H^{\prime}$. Using $H^{\prime}$ ' (instead of $H$ ) and setting $\varepsilon=0$, it is possible to calculate osmotic pressures as a function of nanocrystal concentration with the same accuracy as that for the results shown in Fig. 7. Osmotic pressures calculated with $H$ and $\varepsilon$ are very nearly the same as those calculated with $H^{\prime}$ and $\varepsilon=0$. 


\section{Conclusions}

Number-average molecular weight and osmotic second virial coefficients are reported for quasi-spherical CdSe nanocrystals of size range 2 to $6 \mathrm{~nm}$ in toluene in the range 31 to $45^{\circ} \mathrm{C}$. Experimental molecular weights agree reasonably with calculations considering a CdSe nanocrystal as a sphere of bulk material with a surface coverage of TOPO polymer molecules. The osmotic second virial coefficient for CdSe nanocrystals in toluene rises with solution temperature and falls with nanocrystal molecular weight. The theta temperature is about $35^{\circ} \mathrm{C}$.

Our experimental results indicate that molar absorption for CdSe nanocrystals increases with particle diameter. Our results suggest that the oscillator strength slightly decreases with decreasing particle size.

Experimental osmotic pressures are represented with a perturbed hard-sphere equation of state. The perturbation was approximated by a (relatively) long-range (Hamaker) attraction and a (relatively) short-range interaction due to adsorbed polymers. The longrange attraction was described by the Hamaker approximation for semiconductors in toluene solutions while the short-range interaction was adjusted to fit the experimental data. Our results show that at the experimental conditions considered here, the shortrange interaction can be repulsive or attractive, depending on the sample and on solution conditions. The repulsion is likely due to entropic reasons, while the attraction is probably due to bridging of TOPO polymer brushes between nanocrystals or to partial TOPO coverage of the CdSe nanocrystals.

\section{Acknowledgments}

TEM experiments were done either at EML, University of California, Berkeley or at NCEM, Lawrence Berkeley National Laboratory, Berkeley. This work was supported in part by the Director, Office of Science, Office of Basic Energy Sciences, Chemical Science Division of the U.S. Department of Energy under Contract Number DE-AC0376SF00098. D.Z. thanks the Fundação de Amparo à Pesquisa do Estado de São Paulo (FAPESP) for a fellowship under contract number proc. 99/08603-7. 


\section{References}

1. M. Bawendi, Solid state Commun. 107 (1998) 709. A.P. Alivisatos, Science 271 (1996) 933.

2. J. Hu, L. Li, W. Yang, L. Manna, L. Wang, A.P. Alivisatos, Science 292 (2001) 2060.

3. C.-C. Chen, C.-Y. Chao, Z.-H. Lang, Chem. Mat. 12 (2000) 1516-1518. L. Manna, E.C. Scher, A.P. Alivisatos, J. Am. Chem. Soc. 122 (2000) 12700.

4. V.F. Puntes, K.M. Krishnan, A.P. Alivisatos, Science 291 (2001) 2115.

5. M. Bruchez Jr, M. Moronne, P. Gin, S. Weiss, A.P. Alivisatos, Science 281 (1998) 2013.

6. W.C.W. Chan, S. Nie, Science 281 (1998) 2016. S. Pathak, S.-K. Choi, N. Arnheim, M.E. Thompson, J. Am. Chem. Soc. 123 (2001) 4103-4104.

7. M. Kuno, J.K. Lee, B.O. Dabdonsi, F.V. Mikulec, M.G. Bawendi, J. Chem. Phys. 106 (1997) 9869.

8. C.B. Murray, D.J. Morris, M.G. Bawendi, J. Am. Chem. Soc. 115 (1993) 8706.

9. K.J. Bowen-Katari, V.L. Colvin, A.P. Alivisatos, J. Phys. Chem. 98 (1994) 4109.

10. O. Schmelz, A. Mews, T. Basche, A. Herrmann, K. Mullen, Langmuir 17 (2001) 2861.

11. D. Rosenbaum, P.C. Zamora, C.F. Zukoski, Phys. Rew. Lett. 76 (1996) 150.

12. S. Ramakrishnan, C.F. Zukoski, J. Chem. Phys. 113 (2000) 1237.

13. J. M. Prausnitz, R.M. Lichtenthaler, E.G. de Azevedo, Molecular Thermodynamics of Fluid-Phase Equilbria, $3^{\text {rd }}$ Ed., Prentice Hall, N.J., 1999.

14. M.P. Tombs, A.R. Peacocke, The Osmotic Pressure of Biological Macromolecules, Claredon Press, Oxford, 1974.

15. J.J. Shiang, V. Kadavanich. R.K. Grubbs, A.P. Alivisatos, J. Phys. Chem. 99 (1995) 17417.

16. Y. Wang, Y. Wang, Y. Wang, K. Huang, T. He, F.C. Liu, Spectrochim. Acta 50A (1994) 2203.

17. T. Vossmeyer, L. Katsikas, M. Giersig, I.G. Popovic, K. Diesner, A. Chemseddine, A. Eychmuller, H. Weller, J. Phys. Chem. 98 (1994) 7665.

18. T. Rajh, O.I. Micic, A.J. Nozik, J. Phys. Chem. 97 (1993) 11999.

19. W.B. Russel, D.A. Saville, W.R. Schowalter, Colloidal Dispersion, Cambridge University Press, Cambridge, 1989.

20. J.M. Israelachvili, M. Tirrell, J. Klein, Y. Almog, Macromolecules 17 (1984) 204209.

21. J. Israelachvili, 'Intermolecular and Surface Forces', $2^{\text {nd }}$ Ed., Academic Press, London, 1992.

22. H. Mattoussi, A.W. Cumming, C.B. Murray, M.G. Bawendi, R. Ober, Phys. Rev. B 58 (1998) 7850.

23. L. Bergstrom, Adv. Coll. Int. Sci. 70 (1997) 125-169.

24. N.F. Carnahan, K.E. Starling, J. Chem. Phys. 53 (1970) 600. 
Table 1: Experimental results from membrane osmometry, TEM analysis, and UV-vis experiments. $\sigma$ is the nanocrystal diameter determined by TEM; $T$ is the experimental temperature; $M_{n}$ is the number-averaged molecular weight from membrane osmometry; $B$ is the osmotic second virial coefficient; $\gamma$ is the molecular extinction coefficient in units of $\left(\mathrm{g} / \mathrm{l}^{-1} \mathrm{~cm}^{-1}\right.$, while $\Gamma$ is the molecular extinction coefficient in units of $\mathrm{M}^{-1} \mathrm{~cm}^{-1}$.

\begin{tabular}{c|cccccc}
\hline$\#$ & $\sigma / n m$ & $T /{ }^{\circ} \mathrm{C}$ & $M_{n} /(\mathrm{g} / \mathrm{mol})$ & $B /\left(10^{7} I \mathrm{~mol} / \mathrm{g}^{2}\right)$ & $\gamma /(\mathrm{l} / \mathrm{g} \mathrm{cm})$ & $\Gamma /(\mathrm{l} / \mathrm{mol} \mathrm{cm})$ \\
\hline 1 & 2.4 & 42.4 & 150,000 & $0.50 \pm 0.05$ & 1.84 & 276,000 \\
2 & 2.5 & 42.5 & 175,000 & $0.6 \pm 0.1$ & 2.03 & 355,000 \\
3 & 2.6 & 43 & 169,000 & $0.40 \pm 0.02$ & 2.52 & 426,000 \\
4 & 2.6 & 42.8 & 180,000 & $0.9 \pm 0.1$ & 2.42 & 436,000 \\
5 & 2.7 & 46 & 210,000 & $1.6 \pm 0.1$ & 2.29 & 481,000 \\
6 & 2.8 & 31.3 & 170,000 & $-0.45 \pm 0.1$ & 2.72 & 462,000 \\
7 & 2.9 & 39.4 & 235,000 & $1.0 \pm 0.1$ & 2.44 & 573,000 \\
8 & 3.1 & 42.4 & 470,000 & $0.30 \pm 0.05$ & 2.34 & $1,100,000$ \\
9 & 3.1 & 44.4 & 500,000 & $1.8 \pm 0.8$ & 2.68 & $1,340,000$ \\
10 & 3.8 & 37 & 600,000 & $0.75 \pm 0.05$ & 2.39 & $1,430,000$ \\
11 & 3.8 & 39.2 & 290,000 & $0.12 \pm 0.02$ & 1.55 & 450,000 \\
12 & 3.8 & 39.2 & 290,000 & $0.13 \pm 0.02$ & 1.63 & 473,000 \\
13 & 4.2 & 39.8 & 500,000 & $0.25 \pm 0.05$ & 1.48 & 740,000 \\
14 & 4.3 & 31 & 650,000 & $1.0 \pm 0.1$ & 1.55 & $1,010,000$ \\
15 & 4.6 & 36.5 & 440,000 & $0.7 \pm 0.1$ & 1.6 & 704,000 \\
16 & 4.6 & 37 & 550,000 & $0.15 \pm 0.03$ & 1.51 & 831,000 \\
17 & 4.6 & 42.4 & 460,000 & $0.4 \pm 0.05$ & 1.39 & 639,000 \\
18 & 5.7 & 41.6 & $1,200,000$ & $0.1 \pm 0.02$ & 1.31 & $1,570,000$ \\
19 & 6.1 & 38 & 880,000 & $-0.08 \pm 0.01$ & 1.39 & $1,220,000$ \\
\hline
\end{tabular}


Table 2: Parameters $\sigma, H$, and $\varepsilon$ for Eq.(6) for the osmotic-pressure data presented in Fig.7. When $\varepsilon$ is set equal to zero and $H$ in Eq.(4) is used as the adjustable parameter to fit experimental osmotic pressures, $H^{\prime}$ is obtained. Osmotic pressures calculated in the two approximations are very similar.

\begin{tabular}{c|cccccc} 
Case & $\boldsymbol{M}_{\boldsymbol{n}} \mathbf{g} / \mathbf{m o l}$ & $\boldsymbol{T} /{ }^{\circ} \mathbf{C}$ & $\sigma / \mathbf{n m}$ & $\boldsymbol{H} / \boldsymbol{k T}$ & $\varepsilon / \boldsymbol{k T}$ & $\boldsymbol{H}^{\prime} / \boldsymbol{k T}$ \\
\hline I & 170,000 & 31.3 & 2.8 & 2.85 & -3.6 & 6.00 \\
II & 170,000 & 43.0 & 2.6 & 2.75 & 25.0 & -10.0 \\
III & 150,000 & 42.4 & 2.4 & 2.75 & -1.1 & 3.61 \\
IV & 550,000 & 37.0 & 4.6 & 2.80 & 25.0 & -10.0 \\
V & 880,000 & 38.0 & 6.1 & 2.79 & -2.6 & 5.04
\end{tabular}



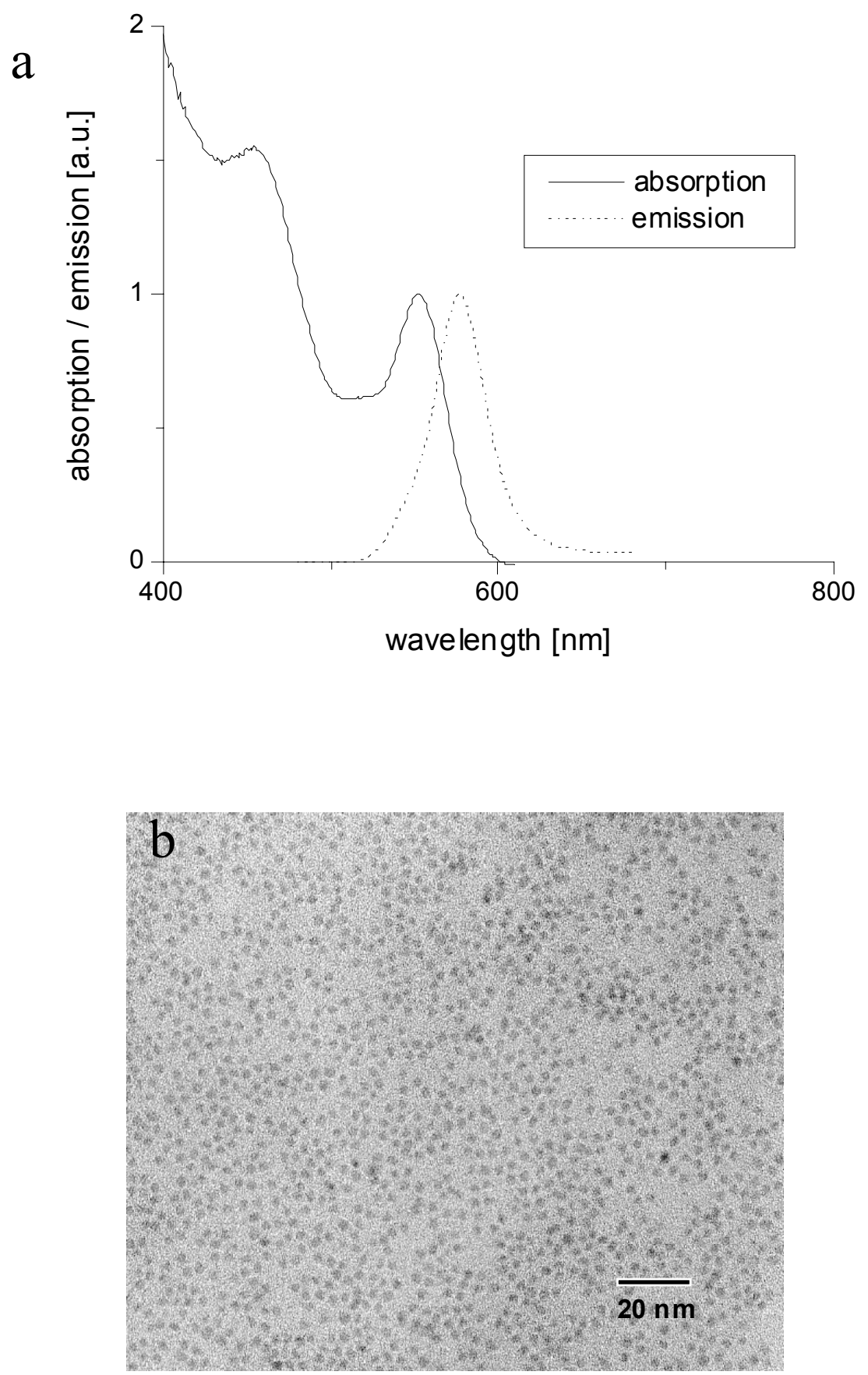

Figure 1: Typical UV adsorption/emission spectrum for a solution of CdSe nanocrystals in toluene (Fig.1a), and TEM image of a CdSe sample used in this work (Fig.1b). 


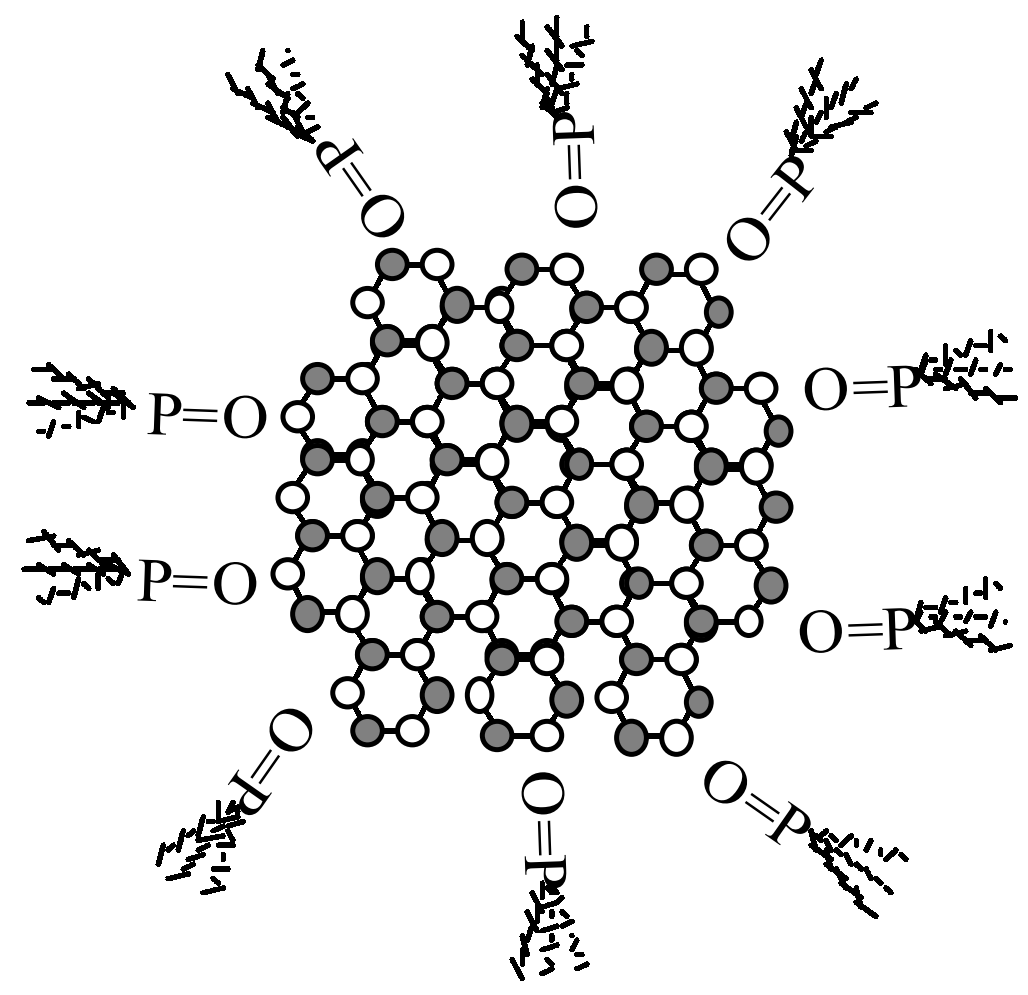

Figure 2. Schematic representation of a CdSe nanocrystal considered in this work. Because of the synthetic route followed to its preparation, a CdSe nanocrystal is characterized by a semiconductor core surrounded by TOPO chains anchored to its surface. 


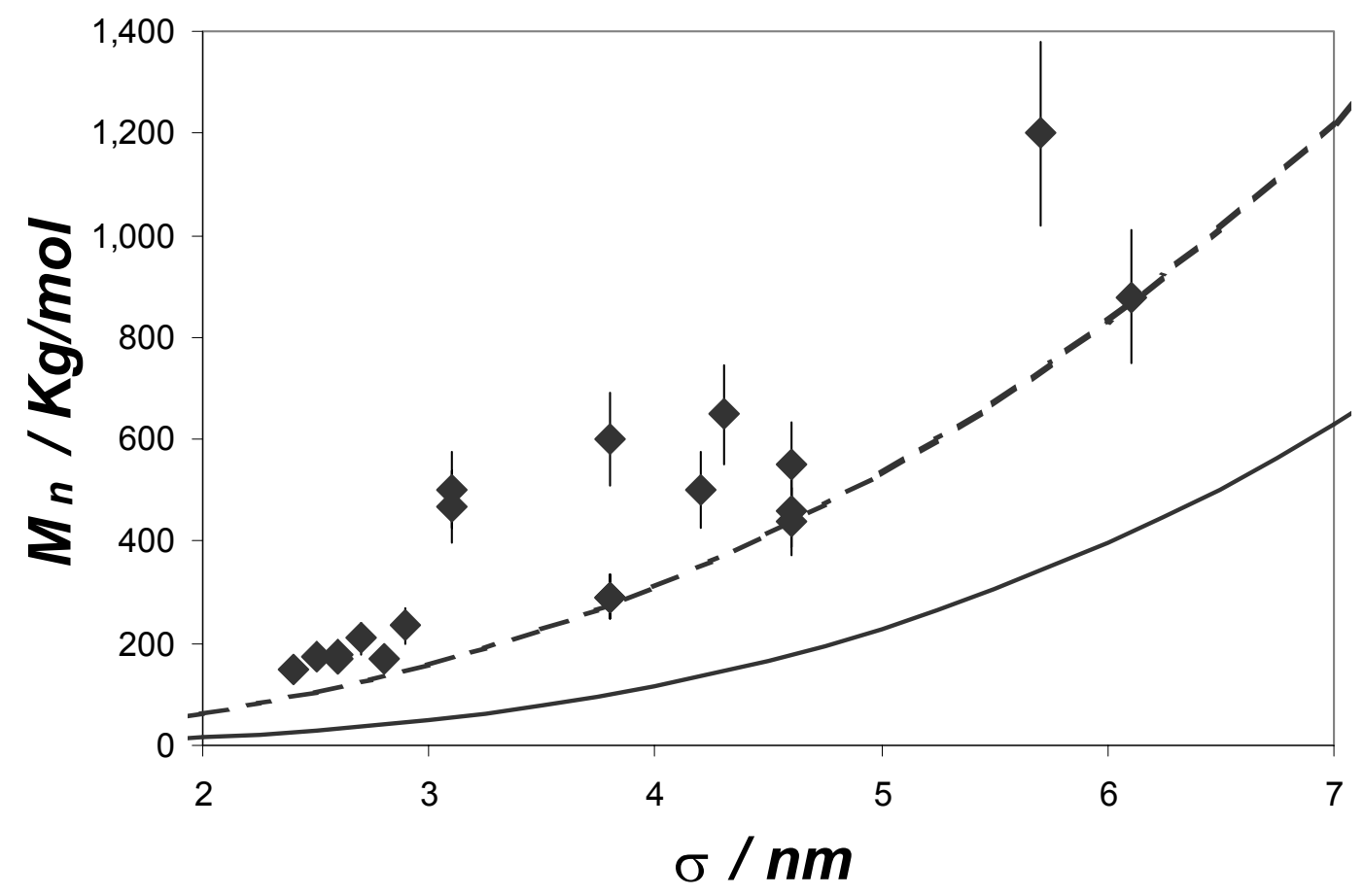

Figure 3: Experimental (points) molecular weight as a function of nanocrystal diameter. The solid line gives theoretical molecular weights computed assuming that the density of each nanocrystal is equal to that of bulk CdSe semiconductor $(5.81 \mathrm{~g} / \mathrm{cc})$. The broken line gives a theoretical molecular weight computed assuming that each $\mathrm{CdSe}$ nanocrystal is uniformly coated with 10 TOPO polymer molecules per $\mathrm{nm}^{2}$ of the semiconductor surface. 


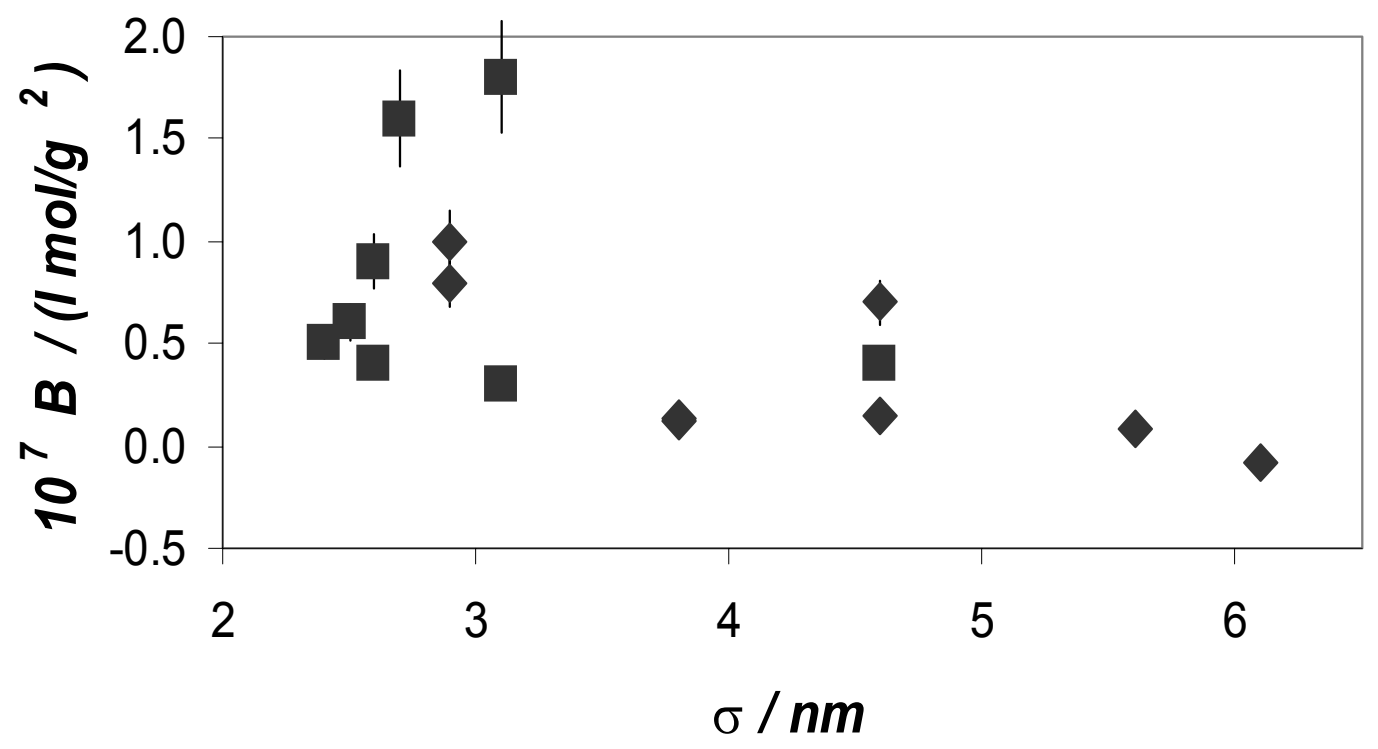

Figure 4: Osmotic second virial coefficient as a function of nanocrystal diameter for the nanocrystals considered in this work in dilute solution in toluene. Diamonds are for nanocrystals in toluene solutions at about at $38^{\circ} \mathrm{C}$, squares are for nanocrystals in toluene solutions at about $42^{\circ} \mathrm{C}$. 


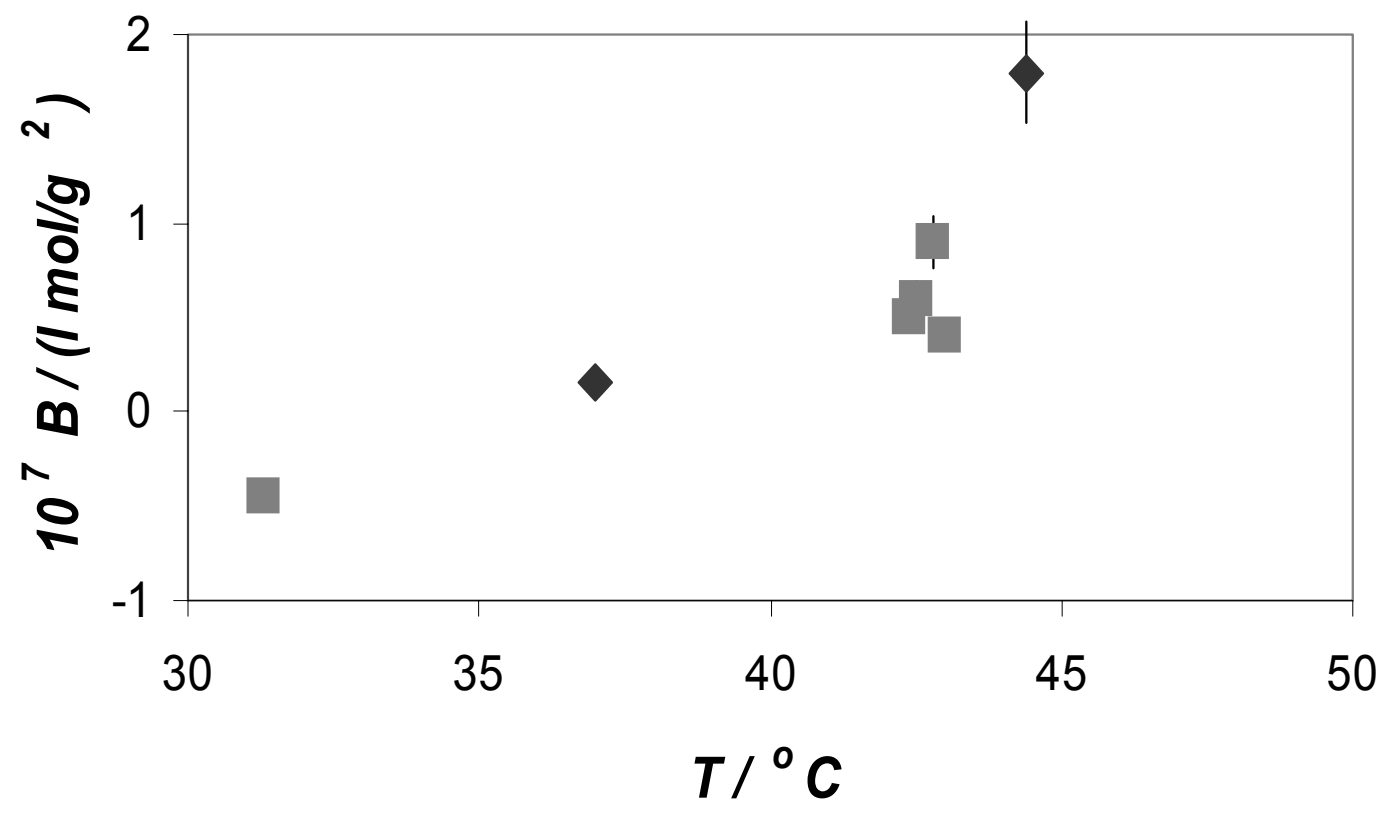

Figure 5: Experimental osmotic virial coefficients for nanocrystals in toluene as a function of experimental temperature. Diamonds are for nanocrystals with molecular weight about 500,000 g/mol; squares are for nanocrystals with about 170,000 g/mol molecular weight. 


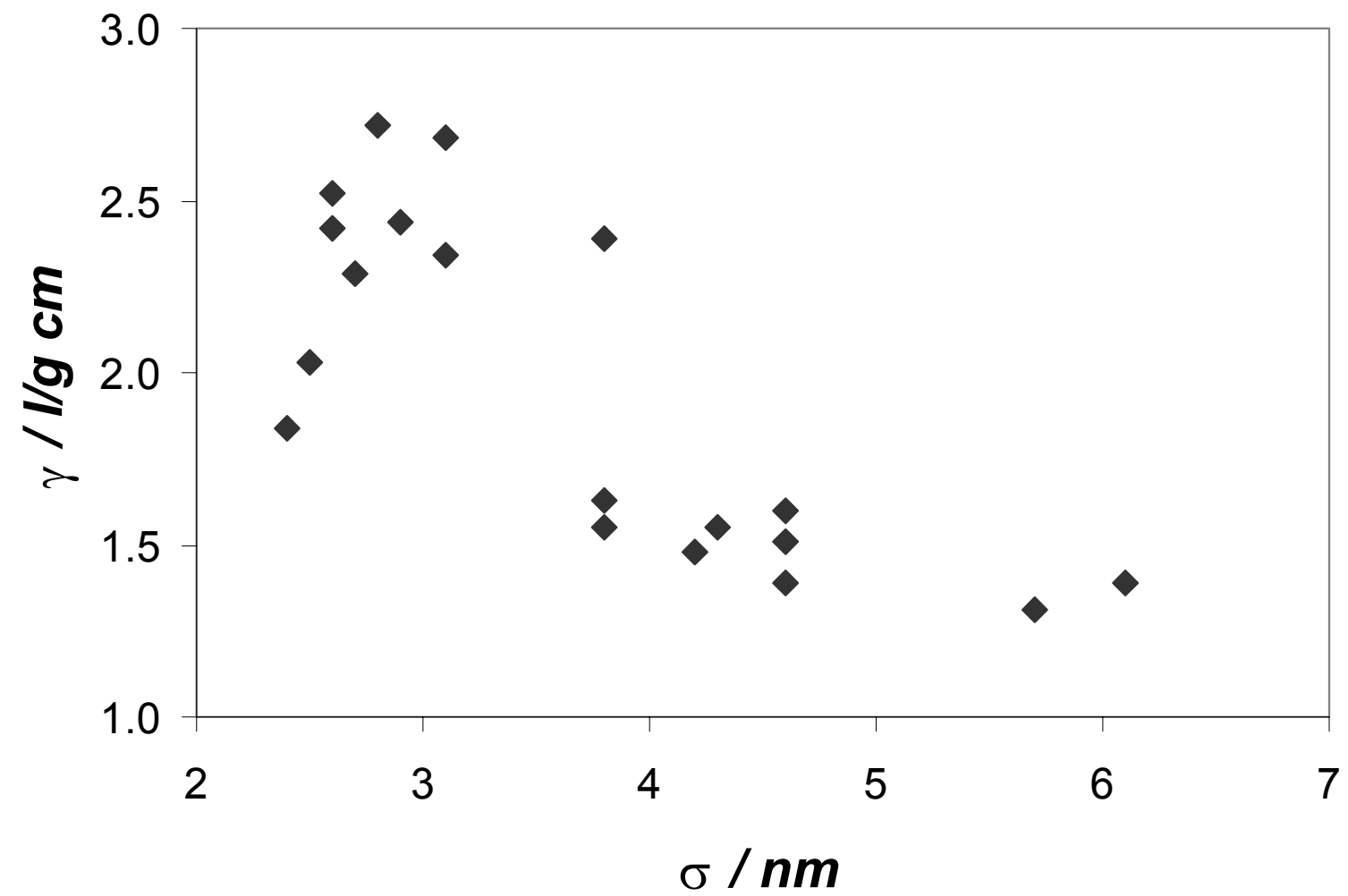

Figure 6. Molecular extinction coefficient, $\gamma$, as a function of nanocrystal diameter. 


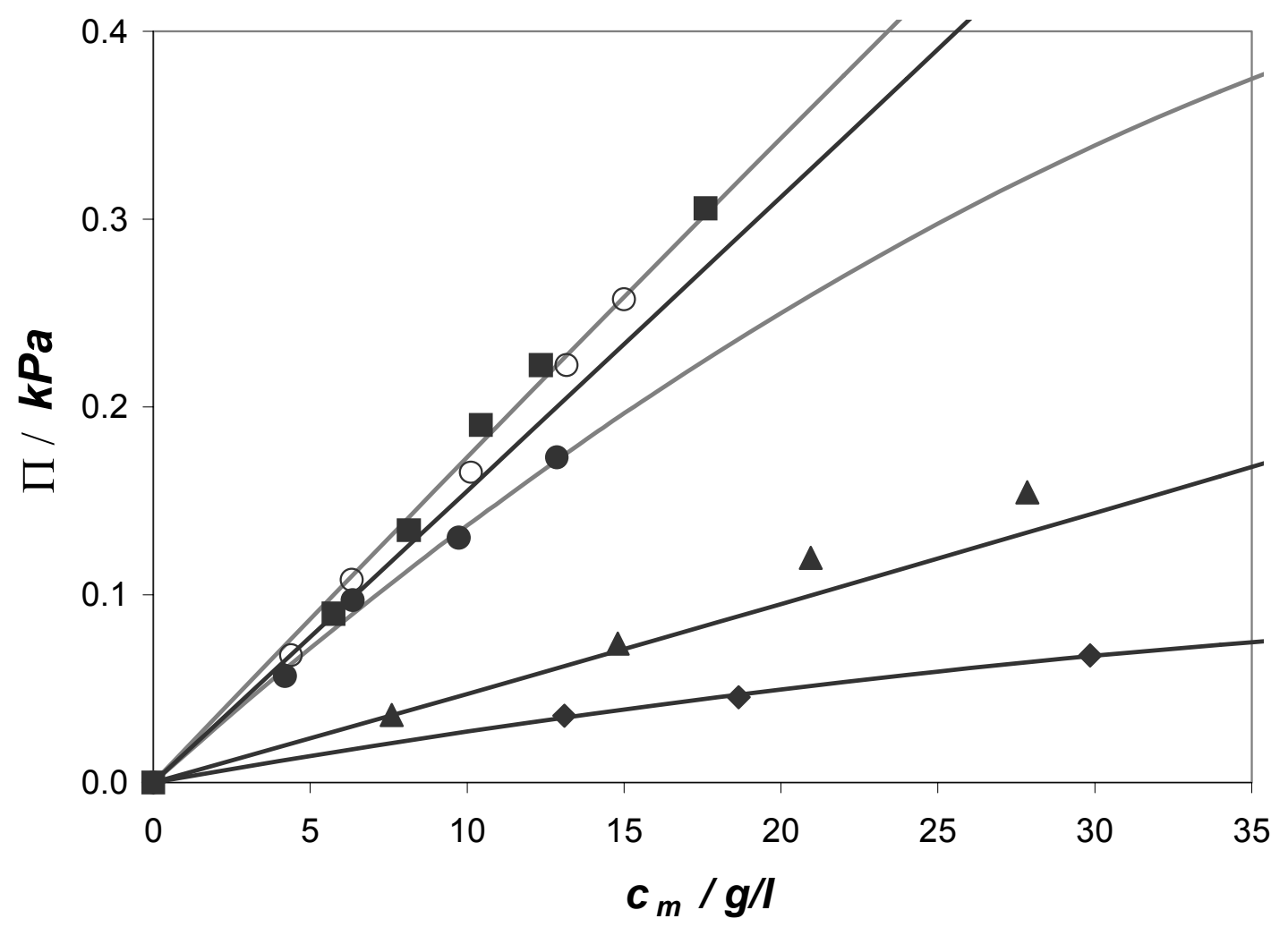

Figure 7: Experimental and calculated osmotic pressure data for nanocrystals in toluene. Full circles are for case I nanocrystals in Table 2, empty circles are for case II, squares are for case III, triangles are for case IV, and diamonds are for case V in Table 2. Lines are obtained by Eq.(6) using parameters $\sigma, H$, and $\varepsilon$ given in Table 2. 